\title{
A 28-year-old man with acute chest pain
}

\section{Sean A. Kennedy, Sheldon M. Singh MD}

A 28-year-old man presents to the emergency department with a five-hour history of retrosternal chest pain radiating to his left arm. He reports that the pain is worse on inspiration and eases when he leans forward. His past medical history is unremarkable. The patient denies recent use of medications or recreational drugs. On examination, he is sweating and is in considerable pain. No other important findings are observed on physical examination; most notably, a pericardial friction rub is not heard. The attending physician suspects acute pericarditis.

\section{What is the cause of this patient's pericarditis?}

Potential causes of pericarditis are numerous and include trauma, viral infection, tuberculosis, malignant disease and autoimmune conditions. Observational studies in developed countries have shown that the search for causative factors is often fruitless, because the condition is deemed to be idiopathic in more than $80 \%$ of patients. ${ }^{1}$ Symptoms of pericarditis that do not improve after one to two weeks probably indicate causes other than viral or idiopathic ones. ${ }^{2}$

\section{What investigations should be considered?}

At least two of the following four criteria should be present for a diagnosis of acute pericarditis: characteristic chest pain; pericardial friction rub; pericardial effusion; and typical electrocardiogram (ECG) changes (widespread upward and concave ST-segment elevation and PR-segment depression, except in the aVR lead, which may show an elevated PR segment). ${ }^{2}$ A pericardial friction rub can be detected in $85 \%$ of patients at some point during the course of the condition. It is best heard at the left lower border of the sternum, and it can be triphasic, biphasic or monophasic. ${ }^{2}$
To make an accurate diagnosis and assess for complications in patients with suspected pericarditis, guidelines recommend that an ECG, a transthoracic echocardiogram and a chest radiograph be obtained, as well as measurement of inflammatory markers (leukocyte count, Creactive protein level and erythrocyte sedimentation rate) and markers of myocardial injury (cardiac troponin levels and creatine kinase level). ${ }^{2}$ Other common causes of pleuritic chest pain must be ruled out, including cardiac ischemia, pneumonia, costochondritis, pulmonary embolism and substance abuse (notably cocaine). ${ }^{2}$

An echocardiogram should be ordered urgently to assess myocardial damage if troponin levels are elevated or there are signs of a large pericardial effusion or cardiac tamponade (e.g., muffled heart sounds, hypotension, pulsus paradoxus or substantial cardiomegaly on chest radiograph). ${ }^{2}$ Otherwise, the echocardiogram could be ordered on an outpatient basis. ${ }^{2}$

Patients who meet the diagnostic criteria for pericarditis and have elevated troponin levels or new left ventricular systolic dysfunction on echocardiography can be considered to have myopericarditis, or inflammation of both the myocardium and pericardium. Myopericarditis follows a relatively benign course, and patient outcomes are similar to those for pericarditis at one year. ${ }^{3}$

Coronary angiography may be considered in patients in whom it is difficult to distinguish coronary artery disease from pericarditis. These are most often patients with elevated troponin levels or major risk factors for coronary artery disease, such as smoking, diabetes, obesity, and a history of myocardial infarction, stroke or peripheral arterial disease. ${ }^{2}$

Pericardial biopsy and pericardiocentesis are seldom indicated for diagnosis. These should be performed only when the pericarditis is refractory to treatment or is associated with large pericardial effusion (> $20 \mathrm{~mm}$ on echocardiogram) or cardiac tamponade, or when malignant disease or tuberculosis is suspected. ${ }^{2}$
Competing interests: None declared.

This article has been peer reviewed.

Correspondence to:

Sean Kennedy, sean.kennedy@medportal.ca

CMAJ 2014. DOI:10.1503 /cmaj.131252 


\section{Does this patient need to be admitted to hospital?}

Two prospective observational studies identified several factors predictive of a poor prognosis in patients with acute pericarditis (Box 1). ${ }^{4,5} \mathrm{~Pa}$ tients with one or more of these factors were at increased risk of recurrence, tamponade and constrictive pericarditis. Therefore, it is reasonable to consider hospital admission for this group. ${ }^{4,5}$

\section{What treatments should this patient receive?}

Treatment is primarily directed at reducing the severity and duration of symptoms and the risk of recurrence. Treatment is typically given for one to two weeks if a first episode and for two to four weeks if a recurrent episode, with tapering over three months. ${ }^{2}$ Strong evidence exists to support treatment of acute and recurrent pericarditis with colchicine in addition to a high-dose regimen of acetylsalicylic acid (ASA). In two randomized controlled trials, patients who were given both ASA (800 mg orally four times daily, with tapering over one month) and colchicine (1 mg orally twice daily for 24 hours followed by $0.5 \mathrm{mg}$ once daily for three months) had substantially faster symptom resolution and lower recurrence rates than patients given ASA alone. ${ }^{6,7}$ Common adverse effects of colchicine include diarrhea, nausea, vomiting and fatigue. Serious adverse effects are rare but may include myelosuppression and liver dysfunction. ${ }^{6.7}$

If colchicine cannot be taken because of poor adherence to treatment or adverse effects, use of a nonsteroidal anti-inflammatory drug (NSAID) alone has been shown to alleviate symptoms substantially. ${ }^{6.7}$ No studies to date have directly compared ASA with other NSAIDs in the treatment of

\section{Box 1: Indicators of a poor prognosis of} acute pericarditis ${ }^{4,5}$

- Fever

- History of trauma

- Elevated troponin levels

- Subacute course

- Large pericardial effusion

- Cardiac tamponade

- No relief of symptoms after one week of treatment with acetylsalicylic acid or other nonsteroidal anti-inflammatory drug

- History of oral anticoagulant use

- Immunosuppression (e.g., due to HIV infection, current chemotherapy, current corticosteroid use, cancer, asplenia, renal failure, chronic liver disease, diabetes) acute pericarditis. Either can be used effectively, although ibuprofen is typically recommended. ${ }^{2}$

Glucocorticoids should seldom be used to treat acute pericarditis because of a high risk of recurrence after treatment is stopped. ${ }^{2}$ Current guidelines recommend that glucocorticoids be used only for autoimmune pericarditis, connective tissue disease, uremic pericarditis or pericarditis that is refractory to NSAID therapy. ${ }^{2}$

In patients with other identifiable causes of pericarditis, such as tuberculosis or malignant disease, treatment should be targeted at the underlying condition. ${ }^{2}$

\section{Case revisited}

On initial presentation, the patient's ECG showed widespread ST-segment elevation, and an echocardiogram showed a small pericardial effusion and normal myocardial contractility. His C-reactive protein level and erythrocyte sedimentation rate were elevated; however, the troponin and creatine kinase levels were within normal limits. The patient was deemed to have acute pericarditis. He was discharged with a prescription for ASA ( $800 \mathrm{mg}$ orally four times daily with tapering over one month) and colchicine (1 mg orally twice daily for 24 hours followed by $0.5 \mathrm{mg}$ once daily for three months).

At one-week follow-up, the patient's symptoms had resolved and his C-reactive protein level was normal. At one month, the C-reactive protein level, and the ECG and echocardiogram findings were normal. The colchicine treatment was continued for an additional two months.

\section{References}

1. Zayas R, Anguita M, Torres F, et al. Incidence of specific etiology and role of methods for specific etiologic diagnosis of primary acute pericarditis. Am J Cardiol 1995;75:378-82.

2. Task Force on the Diagnosis and Management of Pericardial Diseases of the European Society of Cardiology. Guidelines on the diagnosis and management of pericardial diseases executive summary. Eur Heart J 2004;25:587-610.

3. Imazio M, Cecchi E, Demichelis B, et al. Myopericarditis versus viral or idiopathic acute pericarditis. Heart 2008;94:498-501

4. Imazio M, Cecchi E, Demichelis B, et al. Indicators of poor prognosis of acute pericarditis. Circulation 2007;115:2739-44.

5. Imazio M, Demichelis B, Parrini I, et al. Day-hospital treatment of acute pericarditis: a management program for outpatient therapy. J Am Coll Cardiol 2004;43:1042-6.

6. Imazio M, Bobbio M, Cecchi E, et al. Colchicine in addition to conventional therapy for acute pericarditis: results of the COlchicine for acute PEricarditis (COPE) trial. Circulation 2005;112:2012-6.

7. Imazio M, Bobbio M, Cecchi E, et al. Colchicine as first-choice therapy for recurrent pericarditis: results of the CORE (COlchicine for REcurrent pericarditis) trial. Arch Intern Med 2005;165:1987-91.

Affiliations: School of Medicine (Kennedy), McMaster University, Hamilton, Ont.; Schulich Heart Centre (Singh), Sunnybrook Health Sciences Centre, University of Toronto, Toronto, Ont.

Contributors: Both authors contributed substantially to the drafting and revising of the manuscript and approved the final version submitted for publication. Both authors agreed to act as guarantors of the work. 